Nouveaux cahiers de la recherche en éducation

\title{
Aider les élèves à apprendre à comparer des nombres décimaux
}

\section{Éric Roditi}

Volume 10, numéro 1, 2007

Apprendre et former : pour quelles réussites scolaires?

URI : https://id.erudit.org/iderudit/1016855ar

DOI : https://doi.org/10.7202/1016855ar

Aller au sommaire du numéro

Éditeur(s)

Faculté d'éducation, Université de Sherbrooke

ISSN

1911-8805 (numérique)

Découvrir la revue

Citer cet article

Roditi, É. (2007). Aider les élèves à apprendre à comparer des nombres décimaux. Nouveaux cahiers de la recherche en éducation, 10(1), 5-26. https://doi.org/10.7202/1016855ar
Résumé de l'article

En s'appuyant sur de nombreux travaux antérieurs menés sur la comparaison des nombres et sur les nombres décimaux, une nouvelle recherche portant sur 402 élèves de 10 à 25 ans ainsi que sur des adultes a permis de mieux comprendre les traitements mis en œuvre dans l'activité de comparaison des décimaux et de repérer des facteurs liés aux difficultés d'apprentissage. Une expérimentation a été menée par une enseignante avec les élèves les plus en difficulté. Elle a montré qu'une aide conduisant les élèves à mettre en relation plusieurs traitements des nombres dans différentes situations et à confronter les raisonnements corrects ou erronés qui justifient ces traitements s'avère une intervention efficace pour qu'ils surmontent leurs difficultés. 


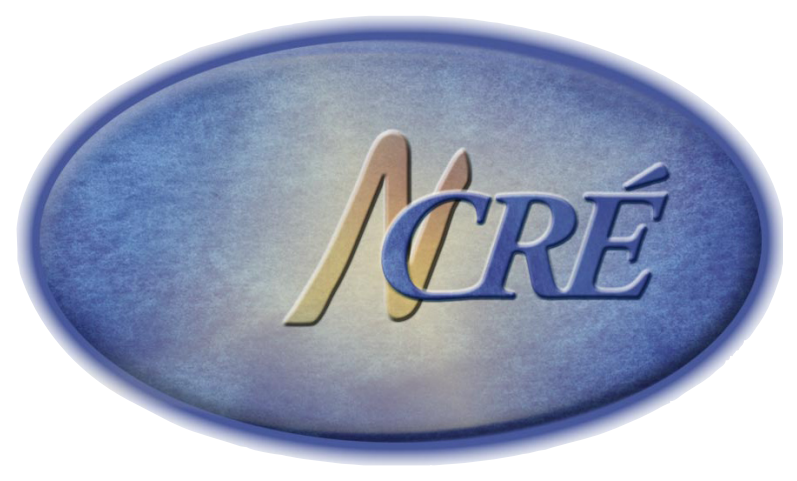

\title{
Aider les élèves à apprendre à comparer des nombres décimaux
}

\author{
Éric Roditi \\ Université Paris Descartes - Sorbonne \\ Laboratoire EDA
}

Résumé - En s'appuyant sur de nombreux travaux antérieurs menés sur la comparaison des nombres et sur les nombres décimaux, une nouvelle recherche portant sur 402 élèves de 10 à 25 ans ainsi que sur des adultes a permis de mieux comprendre les traitements mis en œuvre dans l'activité de comparaison des décimaux et de repérer des facteurs liés aux difficultés d'apprentissage. Une expérimentation a été menée par une enseignante avec les élèves les plus en difficulté. Elle a montré qu'une aide conduisant les élèves à mettre en relation plusieurs traitements des nombres dans différentes situations et à confronter les raisonnements corrects ou erronés qui justifient ces traitements s'avère une intervention efficace pour qu'ils surmontent leurs difficultés.

\begin{abstract}
Based on many previous studies on comparison of numbers and decimal numbers, a new study on 402 students aged 10 to 25 and on some adults allowed for better understanding of the processing approaches implemented in the decimal-number comparison activity and for the identification of the factors associated with learning difficulties. An experiment was conducted by one teacher with the students experiencing the greatest difficulty. It showed that assistance that led students to relate several numbersprocessing approaches to each other in various situations and to take cognizance of the correct or incorrect reasoning underlying these processing approaches was an effective way of helping students overcome their difficulties
\end{abstract}




\section{Introduction}

Les nombres décimaux sont indispensables au citoyen pour connaître ou estimer la valeur d'un bien, la mesure d'une longueur ou d'une surface, etc. Les recherches sur l'enseignement et l'apprentissage des nombres décimaux ont produit des connaissances sur les représentations et les procédures des élèves (Brousseau, 1983; Comiti et Neyret, 1979; Grisvard et Léonard, 1981, 1983 ; Perrin-Glorian, 1986), des ingénieries d'enseignement (Brousseau, 1981 ; Douady et Perrin-Glorian, 1986) et des analyses des pratiques enseignantes (Bolon, 1996; Roditi, 2005a).

Les programmes français d'enseignement ont évolué quant aux nombres décimaux. Leur enseignement n'est plus, comme il l'a été, indépendant de celui des nombres rationnels, avec pour seule représentation celle de l'usage social, ce qui avait pour conséquence de faire obstacle à la conceptualisation. Il n'est pas postérieur à celui des fractions et des nombres rationnels, mais il n'en est pas indépendant car les fractions décimales sont introduites très tôt dans l'enseignement. Les commissions d'élaboration des programmes connaissent les recherches et leurs résultats. Elles les prennent en compte, au moins partiellement. Pourtant, les évaluations nationales à l'entrée dans l'enseignement secondaire (11 ans) montrent des difficultés d'apprentissage persistantes des élèves. En nous appuyant sur des travaux antérieurs, y compris parmi ceux qui concernent la comparaison des nombres entiers, nous cherchons à mieux comprendre les traitements mis en œuvre dans l'activité de comparaison des nombres décimaux. Nous cherchons aussi à identifier, par des analyses croisées, des relations entre les difficultés à effectuer des comparaisons et les difficultés à effectuer d'autres tâches portant sur les nombres. À partir des résultats obtenus, nous nous proposons de concevoir un scénario utilisable par les enseignants pour aider leurs élèves à surmonter leurs difficultés. Ce scénario sera expérimenté et évalué ${ }^{1}$.

Dans la première partie de ce texte, nous indiquons nos cadres de référence ainsi que les résultats des travaux qui nous conduisent à admettre certaines hypothèses quant à la connaissance du nombre, qu'il soit entier ou décimal, mais aussi à en poser de nouvelles, à tester cette fois, au sein d'une problématique générale concernant les difficultés d'apprentissage des nombres décimaux et les aides qui pourraient être apportées aux élèves qui les rencontrent. Dans la deuxième et la troisième partie, nous traitons respectivement des difficultés et des aides: nous spécifions la problématique, explicitons la méthodologie adoptée et indiquons les résultats obtenus. Nous discutons enfin ces résultats pour en montrer la portée et en indiquer les limites, tant à propos de la comparaison des nombres décimaux que du scénario d'aide que les enseignants pourraient adopter.

\section{Cadres et travaux de référence: problématique générale}

\subsection{La double approche didactique et ergonomique des pratiques enseignantes}

La double approche didactique et ergonomique (Robert et Rogalski, 2002, 2005) est un cadre qui permet d'analyser les pratiques enseignantes en tenant compte du fait que ces pratiques visent non seulement l'apprentissage des élèves, mais aussi une réponse à des volontés ou à

1 Cette recherche doit beaucoup à Florence Monfrini-Crépin que nous remercions pour son travail. 
des contraintes personnelles ou professionnelles des enseignants. Les aides que l'enseignant propose en classe font partie de sa pratique professionnelle. Il nous semble indispensable de les considérer au sein de la globalité de sa pratique et en référence à la double approche. Cela nous permet de tenir compte, par exemple, des contraintes de temps qui s'exercent, par le biais des programmes scolaires officiels, sur les choix de l'enseignant tant pour concevoir une séance d'enseignement que pour en gérer son déroulement en classe avec les élèves. En référence à ce cadre théorique, nous admettons aussi que les pratiques sont à la fois complexes et cohérentes et que cela leur confère une grande stabilité après quelques années d'exercice. Nous avons donc choisi, pour proposer de nouvelles formes d'aides aux enseignants, de concevoir ces aides en fonction des résultats concernant les élèves et leur apprentissage, mais aussi avec des enseignants en fonction de leurs pratiques.

Quelques précisions sur ce que recouvre ici l'expression «pratiques enseignantes». Nous en distinguons cinq composantes dans nos analyses. Les composantes cognitive et médiative concernent respectivement les tâches mathématiques proposées aux élèves et les formes de travail effectives avec les élèves (notamment les aides). Les composantes institutionnelle, sociale, personnelle concernent des déterminants des pratiques, intérieurs ou extérieurs à la classe, qui à la fois contraignent et soutiennent l'enseignant dans son travail; il s'agit par exemple, pour illustrer ces trois composantes, des programmes d'étude et des moyens horaires d'enseignement, des normes professionnelles quant à l'organisation de l'enseignement et la gestion d'une classe, des conceptions de l'enseignant quant aux mathématiques, leur apprentissage et leur enseignement.

\subsection{Cadres et travaux concernant l'apprentissage des mathématiques}

Dans la théorie des champs conceptuels, Vergnaud (1990) définit un concept par les situations qui lui donnent du sens (la référence), les invariants sur lesquels repose l'efficacité des schèmes (le signifié), et les formes langagières et non langagières qui lui sont associées (le signifiant). Selon cette théorie, pour tout sujet, le concept de nombre réfère donc aux situations qu'il a rencontrées, situations dont le traitement fait intervenir des nombres. Des situations de dénombrement, de mesure ou de comparaison, ou encore des situations plus complexes conduisant par exemple à composer ou à comparer des mesures de grandeurs et nécessitant d'effectuer des calculs.

Les didacticiens des mathématiques et, notamment, Brousseau (1998) ont renouvelé la notion de situation en lui conférant en particulier une dimension cognitive. Une situation didactique est une situation problématique où le savoir mathématique, éventuellement à construire par l'élève, à adapter ou plus simplement à utiliser, est un moyen de résoudre le problème que pose la situation. Lors de sa construction, le savoir n'est pas reconnu comme tel dans la classe, il est contextualisé. C'est la décontextualisation qui permet d'identifier le savoir indépendamment de la situation didactique qui a permis sa construction en classe. En référence à Brousseau, et en élargissant ces termes à toutes les tâches mathématiques proposées aux élèves, nous distinguons dans ce texte les tâches où les nombres à comparer sont contextualisés de celles où ils ne le sont pas. 
Le cadre de la didactique des mathématiques est notre référence majeure et nous reprenons les hypothèses généralement admises dans ce champ scientifique. Nous supposons que l'apprentissage dépend des situations étudiées, en classe notamment, pour le contenu mathématique qu'elles comportent, mais aussi pour l'organisation de la rencontre entre l'élève et le savoir. Afin d'analyser cette organisation, nous repérons les dynamiques ancien/nouveau (notamment dans le passage des nombres entiers aux nombres décimaux), les dialectiques outil-objet des savoirs (Douady, 1986), les dynamiques contextualisation/décontextualisation (au sens défini précédemment par référence à Brousseau), les registres de représentations des nombres et les changements qui sont proposés (Duval, 1995). Concernant plus précisément les tâches proposées aux élèves nous repérons la responsabilité mathématique qui lui est réservée [ce que Chevallard (1999) désigne par le topo de l'élève] et nous utilisons pour cela les outils d'analyse de tâches développés par Robert (2005). Nous appuyant sur les travaux de Vygotski (1985), nous supposons également que les médiations jouent un rôle important dans l'apprentissage, notamment les aides individuelles ou collectives pour orienter ou réorienter la réflexion des élèves, en distinguant celles qui visent la réalisation de la tâche proposée de celles qui visent directement l'apprentissage, par exemple l'organisation des connaissances mathématiques.

\subsection{Références concernant les nombres et leur apprentissage}

Les premières recherches concernant l'acquisition du nombre par l'enfant ont montré que le nombre se construit à la fois suivant ses deux aspects cardinal et ordinal : à la fois le nombre dit combien et se situe par rapport aux autres nombres. Meljac (2001) explique à ce propos la pensée de Piaget exprimée dans le paragraphe ultime de La genèse du nombre: «le nombre doit être appréhendé comme la synthèse de la relation symétrique (égalité) et des différences (relations asymétriques) s'élabore progressivement grâce à ce que Piaget a appelé l'abstraction réfléchissante» (p. 128). Des travaux plus récents sur l'apprentissage des entiers montrent l'importance de distinguer les aspects sémantiques (valeur) et syntaxiques (notation) (Perret, 1985) en ce qui concerne les formes langagières associées aux nombres. Newman et Berger (1984), d'une part, et DeBlois (1996), d'autre part, ont montré que certains enseignements contribuent à développer respectivement un jugement sur la numérosité, et une idée de distance entre les nombres dans des activités de comparaison. Par ailleurs, Collet (2003) a montré l'influence des systèmes de représentation des nombres (orale, décimale, iconique) sur leur conceptualisation.

Les recherches sur les conceptions et les procédures des élèves quant aux nombres décimaux ont établi que certains élèves, face à des tâches très diverses, produisent des résultats tels que tout se passe comme s'ils traitaient les nombres décimaux comme des couples de deux entiers séparés par une virgule. Constatant, par exemple, que des enfants écrivent 1,38<1,275, Comiti et Neyret (1979) montrent que l'enseignement favorise l'idée selon laquelle les décimaux sont constitués d'une partie entière et d'une partie fractionnaire qui se traitent comme des entiers. Grisvard et Léonard (1981) ont montré que d'autres élèves écriraient au contraire que 1,38 > 1,475 en mobilisant une règle implicite selon laquelle la partie décimale est d'autant plus petite que le nombre de ses chiffres est grand. Brousseau (1980) évoque aussi des erreurs de calcul issues d'un traitement séparé de la partie entière et de la partie décimale comme 2,3:2,3= 4,9. Perrin-Glorian (1986) a montré que certains élèves devant représenter 2,3 mobilisent ce qu'elle nomme une conception «galette» des fractions et des décimaux et représentent une galette circulaire séparée en deux parties par un diamètre horizontal où la partie supérieure est 
partagée en deux et où la partie inférieure est partagée en trois parts, ces parts n'étant même pas équivalentes.

Des travaux menés en psychologie, rappelés par Fayol (1990) dans son livre L'enfant et le nombre, ont montré que lorsqu'un contenu est organisé linéairement par une relation d'ordre (ordre des nombres, des lettres ou des événements), toute tâche de jugement portant sur l'ordre de deux éléments de ce contenu fait apparaître un effet dit de «distance symbolique». Il faut ainsi plus de temps pour comparer 53 et 55 que pour comparer 82 et 55 . Hinrichs, Yurko et $\mathrm{Hu}$ (1981) ont mesuré le temps de réaction et les erreurs commises dans une tâche de comparaison de nombres variables au nombre fixe 55. Ils obtiennent des résultats représentés par le graphique suivant où la courbe représente le temps de réaction et le diagramme en barres représente les taux d'erreurs.

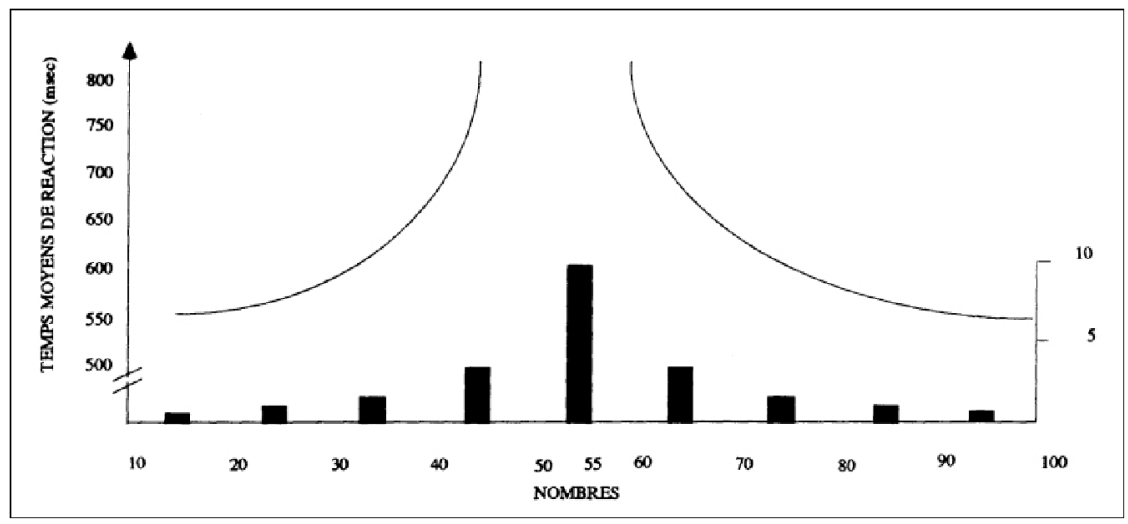

Figure 1 - Comparaison d'un nombre variable au nombre fixe 55: durées moyennes et nombres d'erreurs

De tels résultats montrent que pour comparer deux nombres entiers à deux chiffres, l'activité d'un sujet ne se décrit pas par un algorithme selon lequel on commence par comparer les chiffres de dizaines puis, en cas d'égalité, on compare les chiffres des unités.

Ces travaux et d'autres menés en neuropsychologie ont amené Dehaene et Cohen (1995) à élaborer un modèle anatomo-fonctionnel, appelé «modèle du triple code», selon lequel les nombres sont représentés dans le cerveau par un code visuel permettant la lecture et l'écriture des nombres, un code verbal pour les entendre et les dire, et un code analogique pour en connaître la magnitude. Dehaene et Cohen (Ibid.) interprètent l'effet de distance symbolique entre les nombres par l'utilisation du code analogique. Selon eux, pour comparer les nombres, les sujets mettent en œuvre un traitement sémantique lié à la magnitude et non un traitement syntaxique. Il n'y a pas, à notre connaissance, d'étude qui permettrait de confirmer ou d'infirmer ce type de résultats quant à la comparaison des décimaux.

Ces résultats portent donc sur les procédures mises en œuvre par des personnes qui ont appris à comparer des entiers et pour lesquelles cette activité ne pose pas de problème. Ils peuvent donc être utilisés pour élucider des éléments du fonctionnement normal d'un sujet ou pour faire état de ses difficultés par comparaison avec la norme. Ils ne nous aident pas en revanche à comprendre comment s'acquièrent ces éléments qui constituent ce fonctionnement normal. Dans le modèle du triple code de Dehaene et Cohen (Ibid.), les nombres ne réfèrent en effet ni 
aux situations qu'ils permettent de traiter ni aux schèmes mis en œuvre dans ces traitements; on est loin de la théorie développementale piagétienne.

Ainsi, depuis près d'un siècle, la recherche a accumulé une importante somme de savoirs sur le concept de nombre et sa construction, elle a produit des théories en partie complémentaires et contradictoires. L'analyse et l'interprétation des erreurs, qui sont indispensables pour aider les élèves à surmonter leurs difficultés, dépendent pourtant fortement de ce qui est retenu dans le modèle théorique convoqué pour mener ces analyses et ces interprétations. Comme nous l'avons déjà écrit (Roditi, 2005b), «le chantier important qui conduira à une articulation de ces modèles reste encore ouvert» (p. 51). Cela impose à chaque chercheur d'indiquer précisément ce qu'il retient pour mener ses analyses.

\subsection{Hypothèses et problématique générale de la recherche}

En nous référant aux résultats des travaux que nous avons cités, menés en didactique des mathématiques et en psychologie, nous supposons que l'aspect sémantique des nombres décimaux porte à la fois sur la valeur exacte du nombre et sur ses approximations. Nous admettons que connaître un nombre, c'est connaître non seulement sa valeur avec ses différentes représentations (orale, iconique, numérale et numérique), mais c'est aussi le situer par rapport aux autres, y compris dans des situations où le nombre est une mesure. Dans la réalisation d'une tâche, nous tenons compte à la fois de la production consécutive à la réalisation de la tâche et de la réflexion qui accompagne cette réalisation.

Trois interrogations sont finalement soulevées afin de mieux comprendre les difficultés auxquelles sont confrontés les élèves pour comparer des nombres décimaux. La première interrogation porte sur l'identification de procédures mises en œuvre dans la comparaison des nombres décimaux. Il s'agit notamment de savoir si, comme dans le cas de la comparaison des nombres entiers, on identifie un effet de distance dans les tâches de comparaison des nombres décimaux ou si, dans ce cas précis, le traitement syntaxique de l'écriture décimale est la procédure dominante. La deuxième interrogation est celle de l'évolution favorable ou défavorable des difficultés avec l'âge: les élèves qui ne suivent plus d'enseignement des nombres décimaux progressent-ils spontanément du fait de la diversité des situations sociales qu'ils rencontrent ou, au contraire, les difficultés rencontrées à l'école se renforcent-elles une fois quittée l'école? La troisième interrogation porte sur les relations entre la capacité à comparer les nombres décimaux et la capacité à les reconnaître ou à les représenter avec différentes représentations (numérale verbale ou numérique décimale et fractionnaire) et dans différentes situations, par exemple avec de la monnaie, sur une graduation ou par une fraction de surface.

En fonction des résultats obtenus et de leur interprétation, nous élaborerons un scénario d'aide aux élèves en difficulté qui sera expérimenté et évalué.

\section{Procédures et difficultés dans la comparaison des décimaux}

Dans cette partie, nous étudions les procédures de comparaison des nombres décimaux et les difficultés que rencontrent les élèves. La méthodologie repose sur deux questionnaires, le premier a été proposé à des adultes, le second à des élèves. 


\subsection{Effet distance dans la comparaison des décimaux, interprétations}

En nous inspirant de la tâche de comparaison d'un nombre entier au nombre 55, nous avons conçu une épreuve de comparaison d'un nombre variable décimal à un nombre fixe. Cette épreuve chronométrée a été passée par 40 adultes qui n'avaient pas de difficulté pour comparer des décimaux. Les temps de réponses seront interprétés pour rendre compte d'un éventuel «effet distance» dans cette activité.

\subsubsection{Choix des nombres décimaux}

Nous voulions proposer des nombres décimaux inférieurs ou supérieurs au nombre fixé, sans que la partie entière soit déterminante. Revient-il au même de comparer 19,72 à 19,35 que de comparer 0,72 à 0,35 ? Si c'est la différence absolue entre les nombres qui est le facteur déterminant, ces comparaisons sont équivalentes. Si c'est leur différence relative, alors ces deux comparaisons ne le sont pas. En effet, la différence relative entre 19,72 et 19,35 est inférieure à $2 \%$, alors que celle entre 0,72 et 0,35 est supérieure à $100 \%{ }^{2}$. Cette question relative à la proximité des nombres a déjà été rencontrée, en particulier lors d'une de nos recherches précédentes où nous demandions à des élèves de placer la virgule au produit de deux décimaux en utilisant les ordres de grandeur de ces nombres (Roditi, 2000). Les conclusions laissent penser que les deux comparaisons ne sont pas équivalentes. Pour cette raison, nous avons choisi comme nombre fixe un nombre de partie entière nulle situé approximativement à égale distance de 0 et 1 . Nous n'avons pas choisi 0,5 qui n'a qu'une décimale, nous avons évité 0,55 à cause de la répétition des 5 . Nous avons finalement choisi 0,56 . Il nous a ainsi été possible de le comparer aussi bien à des valeurs supérieures qu'à des valeurs inférieures (valeurs comprises entre 0 et 0,56 ), en faisant varier de manière importante les différences relatives, tout en gardant des nombres ayant la même partie entière.

La liste de nombres à comparer à 0,56 a été élaborée afin de tester l'effet de distance. Nous avons donc choisi des nombres proches et d'autres plus éloignés de 0,56 , par valeur inférieure et supérieure. Nous avons choisi des nombres inférieurs à 0,56 (par exemple, 0,097), puis pour chacun d'entre eux, nous avons adjoint à la liste le nombre symétrique par rapport à 0,56 (avec le même exemple 0,097 , nous avons adjoint 1,023 , car $0,56=(0,097+1,023) / 2)$ ainsi que le nombre dont le rapport à 0,56 est le même que celui de 0,56 au nombre choisi (avec le même exemple 0,097 , nous avons adjoint 3,23, car 3,23/0,56=0,56/0,097). Sans contredire ce principe, nous avons parfois réduit le nombre de décimales pour que les nombres proposés restent comparables quant à leur lisibilité.

\subsubsection{Déroulement de l'épreuve de comparaison}

Quarante adultes âgés de 25 à 60 ans ont répondu au questionnaire: des étudiants et des collègues de l'enseignante qui a participé à la recherche. L'épreuve de comparaison s'est déroulée à l'aide d'un programme informatique qui a été élaboré à cette fin. Comme le montre la copie d'écran ci-après (figure 2), le nombre de référence 0,56 est affiché en permanence. Les nombres à lui comparer apparaissent en dessous. Le sujet doit alors appuyer sur la touche «A» si le nombre qui apparaît est inférieur à 0,56 et sur la touche « $\mathrm{P} »$ si ce nombre est plus grand.

$2(19,72-19,35) / 19,35=0,37 / 19,35 \approx 0,019$ et $(0,72-0,35) / 0,35=0,37 / 0,35 \approx 1,06$. 


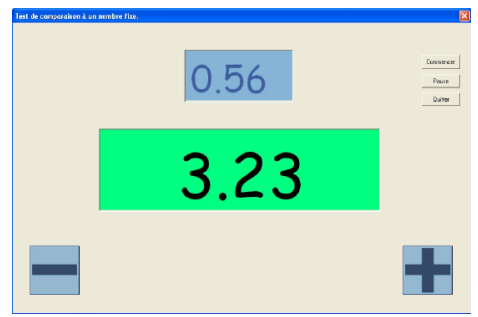

Figure 2 - Comparaison d'un nombre variable au nombre fixe $\mathbf{0 , 5 6}$

Ces touches ont été ainsi choisies parce qu'elles se situent respectivement à gauche et à droite du clavier, conformément à l'orientation conventionnelle de l'axe numérique rappelée à l'écran par les signes «-» à gauche et «+» à droite. Des travaux antérieurs ont montré que la latéralité des sujets est sans influence sur le temps de réponse.

Les nombres apparaissent trois fois chacun au cours de l'épreuve et dans un ordre aléatoire, de manière à ce que la variation des temps de réponse ne soit pas imputable à l'ordre d'apparition des nombres. Le programme mesure le temps (en dixièmes de seconde), entre l'apparition du nombre sur l'écran et la réponse du sujet. Les temps correspondant à des réponses inexactes n'ont pas été retenus.

\subsubsection{Des résultats qui affinent ceux trouvés sur les nombres entiers}

Le graphique de la figure 3 représente des temps de réponse moyens pour chaque nombre proposé. Malgré les irrégularités que nous allons interpréter, l'allure générale confirme 1'existence d'un effet distance dans la comparaison des nombres décimaux: lorsque les valeurs s'éloignent du nombre de référence, les temps de réactions diminuent. Pour proposer des nombres de plus en plus proches de 0,56, il a fallu augmenter le nombre de décimale; en supposant un traitement syntaxique important dans la procédure de comparaison, on pourrait interpréter le graphique en affirmant que le nombre de décimales des nombres à comparer à 0,56 explique 1'allongement du temps de réponse au voisinage de 0,56 . Mais le graphique de la figure 4, qui représente les temps de réponse moyens pour chaque nombre dont la partie décimale comporte trois chiffres, réfute cet argument et conduit à admettre un effet distance.

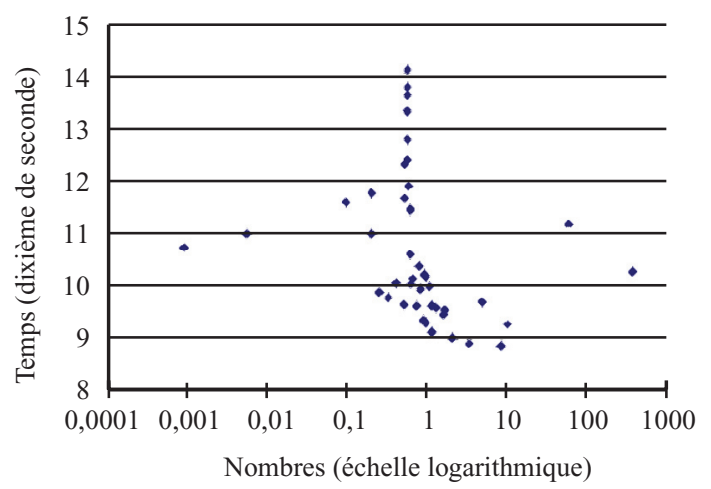

Figure 3 - Comparaison d'un nombre variable au nombre fixe 0,56: durées moyennes 


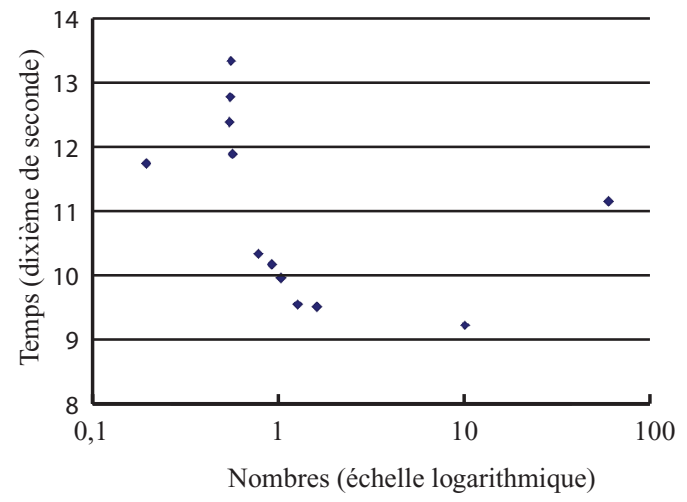

Figure 4 - Comparaison d'un nombre variable à trois décimales au nombre fixe 0,56: durées moyennes

Néanmoins, l'impact du nombre de décimales n'est pas négligeable, il explique certaines irrégularités du graphique. Citons, par exemple, quelques valeurs éloignées de 0,56 où apparaissent, en caractère gras, les décimaux dont le nombre de décimales est différent de deux et pour lesquelles on constate une augmentation du temps de réponse (tableau 1).

Tableau 1

Comparaison d'un nombre variable au nombre fixe 0,56: quelques exemples

\begin{tabular}{|l|l|l|l|l|l|}
\hline Nombres à comparer à 0,56 & 0,64 & 0,72 & 0,784 & 0,8 & 0,87 \\
\hline Temps de réponse $(1 / 10 \mathrm{~s})$ & 10,1 & 9,6 & 10,4 & 9,9 & 9,4 \\
\hline
\end{tabular}

On remarque ensuite que les temps de réponse moyens correspondant au plus grand nombre $(330,11)$, comme aux plus petits $(0,00095$ et 0,0057$)$, sont assez élevés. On peut penser que le nombre de chiffres à lire est un facteur explicatif. On remarque enfin deux irrégularités (entourées dans le graphique ci-contre) qui correspondent aux nombres 4,55 et 55,017. Nous supposons que le «55» figurant dans les écritures chiffrées de ces deux nombres perturbent la comparaison. En effet, bien que 55 soit inférieur à 56, ces deux nombres sont supérieurs à 0,56 . Une recherche complémentaire pourrait étudier de manière plus approfondie l'impact de la perception visuelle des nombres sur la prise de sens (figure 5).

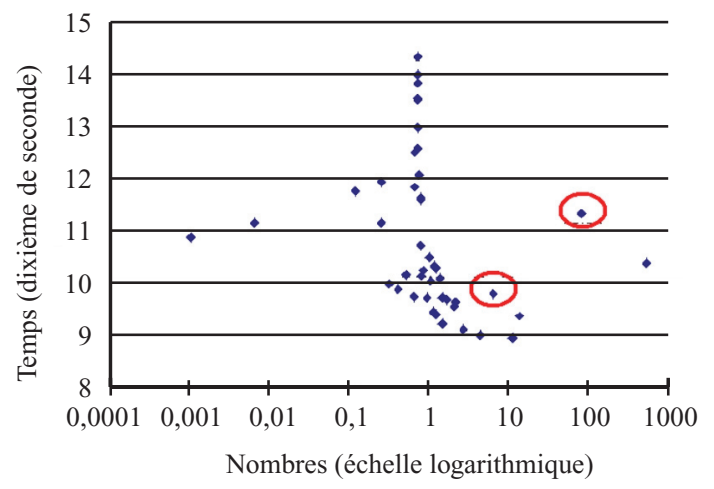

Figure 5 - Effet de l'impact visuel dans la comparaison d'un nombre variable au nombre fixe 0,56 
En conclusion, il apparait que le temps de réponse subit des variations autour d'une tendance générale liée à l'effet distance. Ces variations proviennent de différents facteurs comme le nombre de chiffres des décimaux à comparer ou le nombre de leurs décimales. Le seul traitement sémantique lié à la magnitude repéré dans la comparaison des entiers apparaît comme une conséquence des choix expérimentaux: en restreignant les comparaisons à des nombres entiers à deux chiffres, le facteur issu du traitement syntaxique lié au nombre de chiffres a été fixé. L'effet distance n'est donc pas le seul facteur explicatif du temps de réponse: il apparait plutôt que dans une activité de comparaison, un traitement sémantique est mis en œuvre simultanément à un traitement syntaxique qui permet la lecture des nombres. C'est l'hypothèse que nous adoptons, et qui nous permet de suggérer une piste interprétative des difficultés des élèves : les méthodes enseignées pour comparer les nombres décimaux convoquent souvent exclusivement un traitement syntaxique des écritures décimales, sans doute la plupart des élèves se représentent ce faisant les nombres à comparer de manière suffisante pour effectuer la comparaison, mais certains d'entre eux focalisent sur le traitement syntaxique et se retrouvent en difficulté pour comparer des nombres décimaux.

\section{2 Étude de facteurs de difficulté à comparer des décimaux}

Nous cherchons maintenant à repérer les difficultés rencontrées par les élèves lorsqu'ils ont à comparer des nombres décimaux, et à croiser ces difficultés avec différents facteurs. D'abord, un facteur lié aux élèves, le facteur âge, pour savoir si une fois quittée l'école, la vie courante propose suffisamment de situations où la comparaison de décimaux est nécessaire pour que les élèves acquièrent finalement cette compétence ou si, au contraire, certains élèves restent toujours en difficulté dans cette activité. Ensuite, des facteurs liés aux nombres décimaux et qui ont déjà été soulignés dans les travaux antérieurs : longueur de la partie décimale, le chiffre zéro est ou non le premier chiffre de la partie décimale. Enfin, des facteurs nous sont inspirés par nos choix théoriques, ils concernent notamment les situations qui donnent du sens aux décimaux, et les registres de représentation des nombres.

\subsubsection{Présentation du questionnaire, des élèves interrogés et des premiers résultats}

Cette partie de la recherche repose sur un second questionnaire, cette fois proposé à des élèves. Il porte sur des comparaisons de nombres décimaux dont certaines sont posées dans des contextes de mesure. Il propose aussi des tâches de reconnaissance et de représentation des nombres décimaux. Au total, 51 questions composent le questionnaire, suffisamment faciles pour ne pas décourager les élèves; 39 portent directement sur la comparaison de deux ou de plusieurs nombres décimaux, dont 28 hors contexte, et 12 portent sur les registres de représentation des décimaux. 
Le choix des élèves interrogés est fonction des programmes d'enseignement des nombres décimaux $^{3}$ : introduction des décimaux au CM1 (9 à 10 ans) et approfondissement en $4^{\mathrm{e}}$ (13 à 14 ans) avec les puissances de 10 . Nous avons questionné des élèves de CM2, $6^{\mathrm{e}}$ et $5^{\mathrm{e}}$ (9 à 13 ans pour les élèves sans retard scolaire) ainsi que des élèves de lycée professionnel (16 à 21 ans). Nous n'avons interrogé aucun élève de CM1 ou de $4^{\mathrm{e}}$ pour éviter que les résultats soient influencés par les choix de progression de l'enseignement de leur enseignant. Les élèves de lycée professionnel sont de jeunes adultes, ils ont été choisis afin d'étudier l'effet du facteur âge sur la capacité à comparer des décimaux, sans suivre l'évolution des performances d'une même population durant plusieurs années. L'orientation des élèves en lycée professionnel s'effectue généralement lorsqu'ils sont en difficulté dans l'enseignement secondaire; nous supposons donc que ceux que nous avons interrogés n'étaient pas parmi les élèves les moins en difficulté quand ils avaient de 10 à 12 ans. Au total, 402 élèves âgés de 10 à 25 ans (certains étant en retard scolaire) ont été interrogés dont une centaine par niveau scolaire.

Dans le domaine que nous étudions, la réussite seule n'est pas un critère satisfaisant, puisque des réponses correctes peuvent être obtenues avec des démarches erronées. Indiquons néanmoins que sur les 39 questions de comparaison, le nombre moyen de questions réussies est de 34,2 avec un écart-type de 4,9. Le groupe dont le nombre de réussites est faible ${ }^{4}$ comporte 58 élèves, sa composition est donnée par le tableau suivant (tableau 2).

Tableau 2

Réussites au questionnaire écrit : répartition par niveau scolaire

\begin{tabular}{|l|l|l|l|l|l|}
\hline Niveau & CM2 & $6^{\mathrm{e}}$ & $5^{\mathrm{e}}$ & LP & Ensemble \\
\hline Effectif total & 92 & 117 & 86 & 107 & 402 \\
\hline Groupe faible & 24 & 17 & 11 & 6 & 58 \\
\hline Fréquence relative & $26 \%$ & $15 \%$ & $13 \%$ & $6 \%$ & $14 \%$ \\
\hline
\end{tabular}

Nous avons étudié les effets de différents facteurs sur la réussite aux tâches de comparaison, des facteurs qui portent sur les élèves (leur âge, leur appartenance ou non au groupe faible) ou sur les tâches elles-mêmes (longueur de la partie décimale en référence aux travaux menés par Grisvard et Léonard (1981), nombres donnés oralement ou par écrit, nombres issus d'un contexte ou non). Nous avons aussi croisé les résultats obtenus aux tâches de comparaison avec ceux obtenus aux tâches portant sur la représentation des nombres décimaux dans différents registres.

3 En France, les deux dernières années de l'enseignement primaire sont appelées CM1 (cours moyen $1^{\text {re }}$ année) et CM2 (cours moyen $2^{\mathrm{e}}$ année), elles concernent respectivement des élèves âgés de 9 à 10 ans, et de 10 à 11 ans. La terminologie de cours moyen date d'une époque où ces cours étaient suivis de cours supérieurs destinés aux élèves qui n'étaient pas orientés vers l'enseignement secondaire. L'enseignement secondaire dure sept années. Les six premières années sont numérotées de façon décroissante à partir de la classe de $6^{\mathrm{e}}$ (11 à 12 ans) jusqu'à la classe de $1^{\text {re }}$ (16 à 17 ans), la dernière année est appelée classe de Terminale (17 à 18 ans). Après la classe de $3^{\mathrm{e}}$, certains élèves sont orientés en dehors de l'enseignement secondaire pour suivre un enseignement professionnel.

4 Nombre de réussites inférieur à la moyenne moins un écart-type, c'est-à-dire à 34,2 - 4,9=29,3 


\subsubsection{Effet de l'âge scolaire sur la réussite aux tâches de comparaison}

Afin de rendre compte de l'évolution avec l'âge de la réussite aux tâches de comparaison, nous considérons les quatre groupes d'élèves constitués par le niveau scolaire (CM2, 6e $\left.5^{\mathrm{e}}, \mathrm{LP}\right)$. Le tableau ci-dessous (tableau 3 ) indique l'âge moyen au sein de chaque niveau au moment de la passation du questionnaire et le nombre moyen de réussite aux 39 questions de comparaison.

Tableau 3

Réussite et âge moyen des élèves: répartition par niveau scolaire

\begin{tabular}{|l|l|l|l|l|}
\hline Niveau & CM2 & $6^{e}$ & $5^{\text {e }}$ & LP \\
\hline Âge moyen & 10 ans 7 mois & 11 ans 6 mois & 12 ans 8 mois & 18 ans 10 mois \\
\hline Réussite moyenne $(\mathrm{m})$ & 31,90 & 33,93 & 34,64 & 36,33 \\
\hline
\end{tabular}

On constate que le nombre moyen de réussite est croissant. Un test d'analyse de la variance au seuil de $1 \%$ confirme que les réussites moyennes des quatre groupes sont significativement différentes. Nous concluons donc à une amélioration de la comparaison des nombres décimaux avec l'âge scolaire, et cela bien que les plus âgés aient été choisis dans un type d'établissements qui regroupent des élèves en difficulté d'apprentissage.

Poursuivons l'analyse afin d'évaluer la présence éventuelle d'élèves en difficulté parmi les plus âgés. Nous considérons comme étant en difficulté les élèves de lycée professionnel qui appartiennent au groupe dont le nombre de réussites aux tâches de comparaison est faible. C'est le cas pour 6\% d'entre eux. Ainsi, même si le développement et l'expérience des personnes apparaissent comme des facteurs favorables, ils ne sont pas suffisants pour résorber les difficultés.

\subsubsection{Règles utilisées pour comparer deux décimaux}

Les questions de comparaison de deux décimaux sont posées pour retrouver les résultats des travaux antérieurs et pour les croiser avec deux facteurs : la présence ou non d'un contexte, et l'aptitude à passer d'un registre de représentation des décimaux à un autre. Les «règles implicites » fréquemment utilisées par les élèves pour comparer deux décimaux (dans le cas difficile où les parties entières sont égales et où les parties décimales n'ont pas le même nombre de chiffres) ont déjà été élucidées:

- R1 : le nombre le plus petit est celui dont la partie décimale comporte le moins de chiffres;

- R2: le nombre le plus petit est celui dont la partie décimale comporte le plus de chiffres;

- R3 : si la partie décimale d'un des deux nombres commence par 0, c'est ce nombre le plus petit.

Douze questions portent sur la comparaison de deux décimaux donnés par écrit.

- Six d'entre elles conduisent à l'erreur en cas de mise en œuvre de la règle R1. Pour chacune de ces six questions, le nombre d'élèves commettant une erreur varie de 20 à 35 suivant la question, 
ce qui correspond à $5 \%$ à $9 \%$ des 402 élèves interrogés. Parmi les 58 élèves du groupe dont le nombre de réussites est faible, pour chacune de ces questions, le nombre d'élèves commettant une erreur imputable à $\mathrm{R} 1$ varie de 20 à 35 , ce qui correspond à $34 \%$ à $60 \%$ d'entre eux.

- Trois questions conduisent à l'erreur en cas de mise en œuvre de la règle R2. Pour chacune de ces trois questions, le nombre d'élèves commettant une erreur varie de 11 à 14 suivant la question, ce qui correspond à environ $3 \%$ des élèves. Parmi les 58 élèves du groupe dont le nombre de réussites est faible, pour chacune de ces questions, le nombre d'élèves commettant une erreur imputable à R2 varie de 9 à 11 ce qui correspond à $16 \%$ à $19 \%$ d'entre eux.

- Les trois autres questions portent sur des nombres dont les parties entières étaient différentes.

- Parmi les six questions conduisant à l'erreur en cas d'application de la règle R1, trois questions permettaient de réussir en appliquant la règle R3. Pour suspecter une utilisation de R3 comme amélioration de R1, nous avons déterminé les élèves qui se trompent aux trois questions où R1 conduit à l'erreur et réussissent néanmoins les trois questions où l'application de R3 permet de réussir. On obtient sept élèves $(2 \%)$ dans ce cas, dix $(3 \%)$ si l'on accepte une exception à cette combinaison de réponses et vingt-trois $(6 \%)$ si l'on en accepte deux.

Les résultats confirment donc une utilisation des règles R1, R2 et R3 par certains élèves, pas forcément de manière systématique d'ailleurs. La règle R2 n'a jamais été utilisée par les élèves de lycée professionnel. Tout se passe comme si cette règle se construisait pendant l'enseignement, pour pallier les erreurs commises en utilisant la règle $\mathrm{R} 1$, mais qu'elle disparaissait une fois quittée l'école. En revanche, sur les sept élèves qui utilisent R3 pour améliorer R1, on en trouve quatre en lycée professionnel.

\subsubsection{Effet de la présentation orale ou écrite des nombres décimaux}

En plus des douze questions de comparaison de deux nombres décimaux donnés par écrit, huit questions proposent de comparer deux nombres donnés oralement, par le professeur de la classe et par une simple lecture, par exemple, le nombre 8,14 a été lu «huit virgule quatorze». La consigne donnée aux élèves était toujours de repérer le plus grand des deux. La comparaison des résultats obtenus pour les comparaisons de nombres donnés par écrit avec ceux obtenus pour les comparaisons de nombres donnés oralement montre que la présentation orale accroît la difficulté de la comparaison. Le pourcentage de réponses erronées passe de 5,1\% lorsque les nombres sont donnés par écrit à $11,1 \%$ lorsqu'ils sont donnés oralement. Un test de comparaison des proportions au seuil de $1 \%$, effectué selon la méthode du khi-2, confirme cette différence. Il en est de même pour les 58 élèves du groupe faible: le pourcentage d'erreurs dans ce groupe passe de $29,2 \%$ lorsque les nombres sont donnés par écrit à $41,4 \%$ lorsqu'ils sont donnés oralement.

De ces résultats, nous tirons une hypothèse pour la conception du scénario d'aide à apporter aux élèves en difficulté, les aides ne seront pas données seulement oralement, les discours de l'enseignant seront accompagnés de supports visuels, notamment pour communiquer sur les nombres décimaux. 


\subsubsection{Effet du contexte donnant du sens aux nombres à comparer}

Pour certains décimaux dont la comparaison est demandée hors contexte, des questions portent sur leur comparaison dans un contexte donnant du sens aux nombres. Une question porte par exemple sur la comparaison hors contexte de 14,17 et 14,036; une autre est posée dans un contexte: "Léa et Manu comparent leurs moyennes. Celle de Manu est 14,17 et celle de Léa est 14,036. Qui a la meilleure moyenne?» Une question porte sur la comparaison hors contexte de 1,21 et 1,4; une autre est posée dans un contexte: «Manu mesure 1,21 m. Léa mesure 1,4 m. Qui est le plus grand?». Au total, neuf questions hors contexte sont appariées à neuf questions en contexte.

La variation de réussite aux comparaisons de deux décimaux suivant que la question est posée hors contexte ou en contexte montre que le contexte ne change pas totalement la difficulté rencontrée dans les tâches de comparaison. Néanmoins, on remarque un effet différent suivant que les élèves appartiennent au groupe faible ou non (tableau 4).

\section{Tableau 4}

Effet du contexte sur la difficulté à comparer des décimaux

\begin{tabular}{|c|c|c|c|c|}
\hline & \multicolumn{2}{|c|}{ Nombre d'erreurs } & \multicolumn{2}{c|}{ Pourcentage d'erreurs $^{6}$} \\
\hline & Hors contexte & En contexte & Hors contexte & En contexte \\
\hline Ensemble (402 élèves) & 175 & 240 & $4,8 \%$ & $6,6 \%$ \\
\hline Groupe faible (58 élèves) & 127 & 109 & $24,3 \%$ & $20,8 \%$ \\
\hline Groupe non faible (344 élèves) & 48 & 131 & $1,5 \%$ & $4,2 \%$ \\
\hline
\end{tabular}

Ainsi, les élèves, dans leur ensemble, commettent plus d'erreurs dans les comparaisons en contexte que dans les comparaisons hors contexte. Au contraire, les élèves du groupe faible commettent moins d'erreurs dans les comparaisons en contexte que dans les comparaisons hors contexte. Ce sont donc les élèves les moins en difficulté qui sont le plus gênés par les comparaisons en contexte, pour lesquelles ils commettent près de trois fois plus d'erreurs. Un test d'indépendance selon la méthode du khi-2 au seuil de $1 \%$ confirme que les résultats des élèves du groupe faible sont significativement différents de ceux des autres.

L'interprétation de ces résultats contrastés est difficile, il apparaît que l'accès au sens du nombre ne s'effectue pas de la même façon pour tous les élèves. Deux hypothèses pourraient expliquer cette différence.

- Pour les élèves qui ne sont pas en difficulté, l'écriture suffirait à accéder au sens du nombre (combien il dit et comment il est situé par rapport aux autres) et à effectuer des comparaisons. Le contexte serait alors un ensemble d'informations supplémentaires à gérer, qui pourraient parfois induire en erreur, par exemple lorsque le contexte facilite l'utilisation de règles inexactes. Pour ces élèves, comparer 1,21 et 1,4 est plus facile que comparer 1,21

5 Le nombre de questions «hors contexte» comme «en contexte» est 9; dans la ligne «Ensemble» le nombre d'élèves est 402; le nombre de réponses pour chacune de ces deux catégories est donc $9 \times 402=3618$. Le nombre d'erreurs indiqué est le nombre de réponses erronées parmi les 3618 réponses.

6 Le pourcentage d'erreurs est le quotient du nombre de réponses erronées par le nombre total de réponses obtenues. Ainsi la valeur 24,3\% correspond au rapport: $127 /(58 \times 9)=127 / 522 \approx 0,243$. 
$\mathrm{m}$ et 1,4 $\mathrm{m}$ parce que, comme l'ont montré de nombreux auteurs, le système des unités et des sous-unités induit une utilisation de la règle $\mathrm{R} 1$.

- Pour les élèves du groupe faible, l'écriture seule ne suffirait pas à accéder au sens du nombre (peut-être pour ces élèves est-il difficile de savoir combien il dit, sans savoir de quoi). Le contexte jouerait alors un rôle facilitant, mais pas toujours suffisant pour contrer des règles implicites fortes de traitement syntaxique des écritures numériques. Les réussites ne sont par conséquent pas beaucoup plus nombreuses en contexte que hors contexte.

Dans la perspective d'élaboration d'un scénario d'aide aux élèves en difficulté, nous envisageons de proposer aux élèves des situations facilitant l'accès au sens des nombres et permettant simultanément de confronter ce sens à des traitements syntaxiques des écritures numériques, comme les règles $\mathrm{R} 1$ ou $\mathrm{R} 2$.

\subsubsection{Croisement entre comparaison de deux décimaux et registres de représentation}

Quatre registres de représentation des nombres décimaux figurent dans le questionnaire, deux symboliques et deux graphiques.

Quatre questions proposent de passer de l'écriture à virgule à une écriture sous forme de somme de fractions décimales ou le contraire. Ainsi, par exemple, les élèves devaient changer 7,032 en $7+\frac{3}{100}+\frac{2}{1000}$, et $13+\frac{8}{10}+\frac{3}{100}$ en 13,83 .

Trois questions demandent de lire des nombres décimaux représentés par des marques de graduation sur une droite et de les représenter par leur écriture décimale (figure 6).
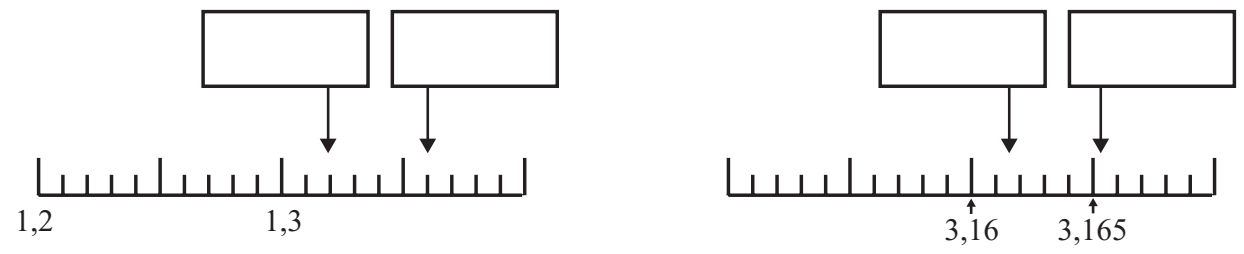

Figure 6 - Représentation des décimaux par des points d'un axe gradué

Dans cinq questions, un carré quadrillé représente l'unité composée de 100 centièmes. Deux questions demandent de lire un nombre décimal représenté par des petits carrés noircis $(0,1$ et $0,01)$, trois questions demandent de représenter des décimaux de manière analogue $(0,3 ; 0,05$ et 0,12 ) (figure 7 ).
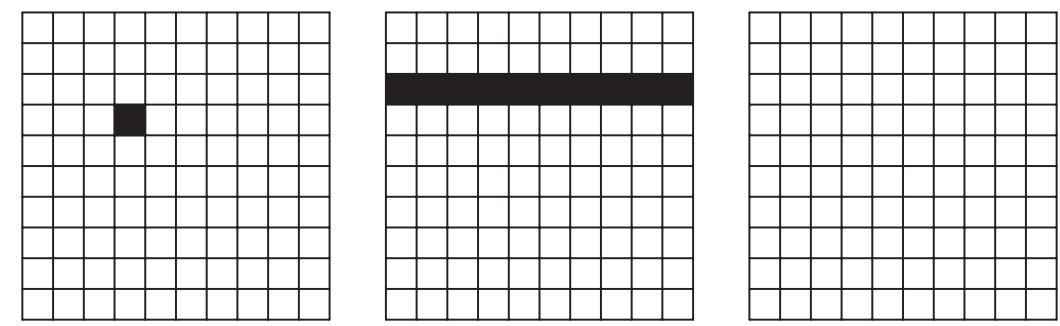

Figure 7 - Représentation d'un décimal par une partie d'un carré quadrillé 
Nous constatons que la capacité à effectuer les changements de registre écriture décimale/ écriture fractionnaire n'est pas indépendante de la capacité à comparer des nombres décimaux : $35 \%$ des élèves qui échouent à ces changements de registres appartiennent au groupe faible, alors que ce groupe ne représente que $14 \%$ de l'ensemble des 402 élèves. Les résultats vont dans le même sens pour le changement de registre écriture décimale/représentation sur une graduation (27\% dans le groupe faible au lieu de $14 \%$ ) et pour le changement écriture décimale/représentation sur un quadrillage ( $23 \%$ dans le groupe faible au lieu de $14 \%$ ). Ces différences de pourcentages sont significatives au seuil de $1 \%$, d'après les tests de comparaison de proportion réalisés selon la méthode du khi-2.

La capacité à comparer les nombres n'est donc pas indépendante de la capacité à les représenter et à changer de registre de représentation. En conclusion de cette étude, trois axes sont retenus pour concevoir un scénario d'aide aux élèves en difficulté pour comparer des décimaux:

- proposer des situations écrites où les nombres décimaux peuvent être lus et pas seulement entendus ;

- favoriser un traitement sémantique des écritures numériques en demandant aux élèves d'exprimer les nombres par différents registres de représentation, et en leur demandant de donner une approximation des nombres utilisés ;

- faire expliciter et critiquer des procédures de comparaison.

\section{Un scénario d'aide aux élèves en difficulté pour comparer des décimaux}

Le suivi et l'expérimentation, par leur professeur de mathématiques, d'un dispositif d'aide aux élèves en difficulté pour comparer des décimaux posent des problèmes méthodologiques importants. Nous avons, pour cette recherche, choisi un autre mode d'expérimentation. Une enseignante intéressée par ces difficultés d'apprentissage et associée à la recherche, a expérimenté le dispositif d'aide avec une partie des 28 élèves de collège qui ont répondu au questionnaire et qui faisaient partie du groupe faible: ceux dont les erreurs aux comparaisons de deux décimaux donnés à l'écrit étaient massives et dont les parents et les chefs d'établissement ont accepté l'expérimentation. Finalement, 11 élèves parmi les 402 ont participé à cette dernière partie de la recherche.

\subsection{Description du scénario}

Les élèves avaient à comparer des décimaux; ils devaient représenter les nombres à comparer, approximativement ou précisément; ils devaient aussi confronter leurs procédures et leurs résultats aux représentations produites, d'une part, et à d'autres procédures correctes ou erronées qui leur étaient soumises, d'autre part. Un post-test a été réalisé pour évaluer l'effet de ces entretiens. Le scénario d'aide s'est déroulé sur deux séances d'une demi-heure chacune en entretiens individuels, le post-test a été réalisé une semaine après. 


\subsubsection{Représentation des nombres décimaux}

La première séance commence par un court questionnaire de comparaisons de deux décimaux; il s'agit pour l'enseignante de travailler avec l'élève à partir de ses productions. Pour chaque réponse produite, l'enseignante engage l'élève à représenter les nombres comparés. Différents supports de représentation sont proposés : des pièces de monnaie (en lien avec les fractions décimales), des graduations, des carrés quadrillés comme ceux proposés dans le questionnaire. En outre, du papier blanc, une règle graduée et une paire de ciseaux sont mis à disposition des élèves pour conduire à la comparaison de longueurs. Afin de motiver la représentation des nombres et, que l'élève l'utilise pour comparer, l'enseignante pose une question à l'élève, en fonction de sa réponse au questionnaire court du début de séance. Ces questions sont toutes prévues dans le scénario.

Lorsque l'élève s'empare du matériel pour représenter les nombres à comparer, l'enseignante suggère de commencer par une approximation. Ainsi, par exemple, pour comparer 8,7 et 8,14 , si l'élève choisit d'utiliser une graduation, l'enseignante incitera l'élève à dire si 8,7 est plus près de 8 ou de 9 , de même pour 8,14 . La réponse de l'élève n'est pas commentée: l'enseignante engage l'élève à poursuivre la représentation.

Voyons maintenant quelles sont les questions posées, c'est-à-dire comment l'enseignante motive la représentation des nombres décimaux et son utilisation pour les comparer.

\subsubsection{Explicitation et critique des règles implicites de comparaison des décimaux}

Poursuivons avec l'exemple de la comparaison de 8,7 et 8,14; 1'élève devait entourer le plus grand des deux nombres. Si l'élève entoure le bon nombre, l'enseignante lui dit: «L'autre jour, un élève comme toi avec qui je travaillais, m'a dit que comme 7 était plus petit que 14, 8,7 était plus petit que 8,14. Qu'est-ce que tu en penses? Comment pourrais-tu faire pour voir qui a raison avec le matériel qui est là?» Si l'élève a entouré le mauvais nombre, l'enseignante cherche d'abord à faire expliciter la procédure de l'élève, puis l'amène à représenter les nombres et à utiliser ses représentations pour raisonner. Si l'élève n'arrive pas bien à expliciter sa démarche, l'enseignante lui suggère la règle implicite qu'il a probablement utilisée: «Un élève m'a expliqué que comme 7 était plus petit que 14, 8,7 était plus petit que 8,14. Qu'est-ce que tu en penses?» Lorsque l'élève reconnaît sa démarche, l'enseignante lui propose une procédure contradictoire: «Un autre élève comme toi avec qui je travaillais $m$ 'a dit que 7 dixièmes c'était pareil à 70 centièmes, et que 70 étant plus grand que 14 , c'était 8,7 qui était plus grand que 8,14. Qu'est-ce que tu en penses? Comment pourrais-tu faire, avec le matériel qui est là, pour voir qui a raison?».

Il ne s'agit donc pas de «refaire» le cours. À aucun moment, elle ne rappelle, les méthodes enseignées pour comparer deux décimaux; au contraire, elle les met en discussion en introduisant son propos par: «L'autre jour, un élève comme toi avec qui je travaillais, m'a dit que...». Ainsi, l'élève ne peut pas préjuger l'exactitude du raisonnement proposé. Pour que l'élève accepte de s'engager dans ces tâches assez complexes, et pour éviter qu'il ne se sente en situation d'échec, l'enseignante choisit de travailler sur les réponses produites sans respecter 
l'ordre du questionnaire et elle commence toujours par une comparaison réussie. Sur le modèle présenté, les interventions de l'enseignante sont prévues dans le scénario et adaptées aux comparaisons et aux règles implicites qui peuvent être mises en œuvre.

Ce scénario est celui de la première séance. Durant la deuxième séance, l'enseignante propose une première série de comparaisons où l'élève doit à chaque fois utiliser le matériel avant de décider lequel des deux nombres est le plus grand, puis une série où le matériel n'est pas disponible, mais où l'enseignante demande à l'élève de se référer à une représentation autre que l'écriture décimale pour justifier sa réponse.

\subsection{Justification du scénario}

Dans sa conception, le scénario d'aide reprend les résultats obtenus grâce aux questionnaires soumis aux adultes et aux élèves. Dans les tâches proposées, les nombres décimaux sont écrits, et ce qui est dit est en relation avec ce qui est écrit. Les consignes données par l'enseignante favorisent un traitement sémantique reposant à la fois sur la recherche de valeurs approchées et sur le changement de registre de représentation. Les questions posées poussent les élèves à expliciter et à critiquer les procédures qu'ils utilisent pour comparer des décimaux.

Un autre type de justification nous semble essentiel: une justification par rapport au travail de l'enseignant. Si les ingénieries didactiques très complètes, construites par Brousseau (1981) ou par Douady et Perrin-Glorian (1986), ne sont pas utilisées dans l'enseignement ordinaire, les manières d'enseigner les nombres décimaux ont tout de même évolué : les programmes ont changé de façon importante et, vingt ans après leur formulation, les propositions de Comiti et Neyret (1979) ont globalement été suivies. Les manuels scolaires sont conformes aux programmes, et le plus souvent, les enseignants les suivent pour concevoir leur enseignement. Pourtant, les difficultés d'apprentissage des élèves persistent. Les causes qui peuvent être évoquées sont nombreuses, l'une d'entre elles nous semble essentielle: changer l'enseignement, c'est changer les pratiques d'enseignement, et les pratiques d'enseignement ne se réduisent pas à leurs composantes institutionnelle et cognitive. La composante médiative est fondamentale : les modalités de travail proposées aux élèves et les interactions verbales en classe comptent énormément dans la relation enseignement-apprentissage. Les pratiques sont aussi personnelles et sociales, elles évoluent lorsqu'un enseignant élargit l'ensemble de ses possibilités d'agir, et pour cela, il faut que l'enseignant ne soit pas seul; c'est ce que nous avons montré dans Roditi (2004).

Nous avons élaboré un scénario d'aide parce que nous avons cherché à proposer aux enseignants des moyens d'intervenir autrement auprès de leurs élèves, tout en restant dans le cadre des programmes actuels d'enseignement des nombres décimaux, à la fois parce que c'est pour eux une obligation liée à leur métier, mais aussi parce que ces programmes semblent aujourd'hui adaptés. La modalité d'aide est particulière, elle n'est pas fréquente dans les classes : la recherche menée par Chapet-Pariès (2004) montre que les échanges verbaux des enseignants avec leurs élèves laissent peu d'initiatives à ces derniers. Autrement dit, nous avons cherché à concevoir des possibles nouveaux pour l'enseignement, en focalisant non pas sur la composante institutionnelle pour élaborer des propositions de modification de programmes ni sur la composante cognitive pour concevoir une nouvelle situation d'enseignement-apprentissage, mais sur la composante médiative pour donner aux enseignants des moyens supplémentaires d'aider leurs élèves lorsqu'ils sont en difficulté. Pour intervenir autrement qu'en expliquant une fois de 
plus la leçon qui n'a toujours pas été comprise, autrement qu'en donnant la réponse ou en la faisant donner par un bon élève, pour intervenir en partant de l'activité de l'élève en difficulté (ce qu'il fait, ce qu'il dit, ce qu'il pense) et en favorisant le développement et l'organisation de ses connaissances sur les décimaux.

Pour terminer cette justification du scénario d'aide, soulignons un dernier argument qui a été un vecteur important dans sa conception. Le lecteur a sans doute pensé que les questions étaient très inspirées des argumentations et contre-argumentations que Piaget (dans Vinh, 1966) luimême proposait dans ses entretiens avec les enfants pour évaluer, par exemple, la conservation des quantités discrètes. Nous ne contestons pas l'inspiration: les connaissances des élèves ne se réduisent pas à ce qu'ils peuvent produire, la pensée qui accompagne cette production est importante, y compris la capacité à prendre en compte les arguments d'autrui, pour évoluer ou pour les réfuter.

\section{3. Évaluation du scénario}

L'enseignante a fait un bilan de ses interventions. Elle retient que la réussite à une comparaison ne prouve pas l'apprentissage et que les explications fournies, même lorsqu'elles sont correctes, sont souvent fragiles lorsqu'elles portent sur l'écriture seulement: «je dois rajouter un zéro avant de comparer» ou encore «le premier chiffre après la virgule est plus grand». Les élèves qui ne tiennent pas compte de la partie entière pour comparer des décimaux rectifient toujours spontanément leur réponse lorsqu'elle leur demande de la justifier. Les raisonnements qui reposent sur la règle $\mathrm{R} 1 \mathrm{du}$ «couple d'entiers » sont clairement explicités par ceux qui se trompent et déstabilisent les autres. En revanche, la règle R2 de la longueur de la partie décimale n'est pas explicitée, mais elle est reconnue par les élèves qui l'utilisent.

En ce qui concerne le recours aux représentations des décimaux, l'enseignante a constaté que l'argent était bien manipulé par les élèves, même si la pièce de 0,1 euro n'est pas une pièce de 1 «décime», mais une pièce de 10 centimes, c'est-à-dire 0,10 euro. En revanche, les représentations graphiques posaient problème pour six des onze collégiens: ils traçaient des segments de $9,4 \mathrm{~cm}$ pour représenter 8,14 en comptant 14 graduations après 8 .

Le post-test proposait 15 comparaisons de décimaux analogues à celles des 12 questions du premier questionnaire que nous considérons ici comme un pré-test. L'évolution éventuelle du nombre moyen d'erreurs permet d'évaluer les effets de l'aide apportée par l'enseignante. Les 11 élèves qui ont bénéficié de l'aide avaient commis 47 erreurs aux 12 questions, ce qui correspond à $35,6 \%$ de réponses fausses. Au post-test, sur les 15 questions, une seule erreur a été commise, cela correspond à $0,6 \%$ de réponses fausses. Les élèves ont donc réalisé un progrès : ils ne font pour ainsi dire plus de faute en comparant deux décimaux. Un test de comparaison de proportion au seuil de $1 \%$ a été réalisé selon la méthode du khi-2, il confirme que la différence de pourcentage est significative. Néanmoins, il est préférable de rester prudent quant à ces résultats pour plusieurs raisons qui tiennent à la méthodologie mise en œuvre. Le post-test a été réalisé seulement une semaine après les séances d'aide, les représentations évoquées lors des entretiens pouvaient être encore très présentes dans l'esprit des élèves. Le déroulement du scénario d'aide a été confié à une seule enseignante, si bien qu'on ne peut exclure la possible influence de certains facteurs personnels qui ne sont pas pris en compte dans la recherche. Bien que tous en grande difficulté et ayant réalisé des progrès importants, le nombre d'élèves 
qui ont suivi le dispositif d'aide est faible et les résultats demandent à être confirmés avec un effectif plus important. Enfin, la transposition en classe du scénario d'aide n'a pas été réalisée, et malgré le soin apporté dans sa conception pour le rendre utilisable par les enseignants, une évaluation dans les conditions ordinaires d'enseignement reste nécessaire.

\subsection{Exploitation possible en classe dans des conditions ordinaires}

Les raisons qui nous ont fait élaborer un tel scénario tiennent aussi à notre analyse du métier d'enseignant et de ses pratiques ordinaires (Roditi, 2005a). Les enseignants interviennent en classe, souvent, et le plus souvent, sous la forme d'un échange direct avec l'élève. Les questions élaborées pour le scénario permettent, d'une part, de laisser l'enseignant prendre des initiatives pour aider ses élèves et permettent aussi, dans la situation de classe, de faire intervenir les autres élèves. L'enseignant peut remplacer «L'autre jour, un élève comme toi avec qui je travaillais, m'a dit que...» par l'intervention d'un autre élève de la classe dont il a repéré à l'avance la production et les arguments. Avec ce type d'interventions, prévues, on échappe au modèle trop caricatural selon lequel le bon enseignant serait celui qui propose une bonne situation, et qui se tait pour laisser les élèves construire leurs connaissances par la seule réalisation des tâches proposées avec la situation. Un modèle que l'enseignant peut tenir un moment, lorsque les élèves se confrontent avec le problème mathématique, un modèle dont l'enseignant peut ainsi sortir avec une réelle alternative au cours magistral.

\section{Conclusions et perspectives}

La procédure de comparaison des nombres décimaux ne repose pas seulement sur un traitement syntaxique de l'écriture décimale, elle repose aussi simultanément sur un traitement sémantique qui met en jeu l'ordre de grandeur des nombres à comparer. Pourtant, l'enseignement propose souvent, comme seule approche, un traitement de l'écriture numérique qui vise à comparer les chiffres de la partie décimale ou à compléter par des zéros pour que les parties décimales des décimaux à comparer aient le même nombre de chiffres. Les élèves en difficulté utilisent des règles implicites inexactes, par exemple traiter la partie décimale comme on traite un entier, ce qui les conduit à affirmer que 3,14 est supérieur à 3,5 au motif que 14 est supérieur à 5. D'ailleurs, lorsque les nombres sont donnés oralement, en disant la partie entière, puis «virgule», puis la partie décimale, les erreurs des élèves en difficulté sont beaucoup plus nombreuses que lorsque les nombres sont donnés par écrit.

La capacité à comparer des décimaux dépend aussi de la qualité de la connaissance de ces nombres. Les élèves qui ne savent pas bien changer de registre de représentation des nombres décimaux se trompent beaucoup plus que les autres. Lorsque les nombres à comparer sont présentés dans une situation, les élèves en difficulté réussissent mieux les comparaisons, comme s'ils utilisaient la situation pour donner du sens aux nombres à comparer. Au contraire, les élèves qui n'ont pas de difficulté ne réussissent pas mieux, même qu'ils seraient parfois gênés pour gérer ces informations supplémentaires. Enfin, les élèves les plus âgés réussissent mieux que les jeunes; la capacité à comparer des nombres décimaux évolue donc favorablement avec l'âge et l'utilisation de ces nombres dans des contextes variés. Cependant, il reste des élèves en difficulté, même parmi les plus âgés, qui ne pourront pas progresser sans une aide spécifique. 
La compréhension des difficultés rencontrées par certains élèves laisse supposer qu'une aide efficace pourrait leur être apportée en les conduisant à changer de registre de représentation des décimaux (monnaie, graduation, etc.) et à mettre en relation ces représentations des nombres et différentes procédures pour comparer les décimaux. Une expérimentation menée par une enseignante avec les élèves de 11-12 ans les plus en difficulté montre qu'un tel travail est efficace. Une expérimentation complémentaire portant sur plus d'élèves, avec plus d'enseignants, réalisée dans les conditions ordinaires de la classe, et avec des évaluations à court, moyen et long terme, reste nécessaire pour confirmer ces premiers résultats.

Bien que cette recherche ait mis au jour des éléments nouveaux sur la compréhension de l'activité de comparaison des décimaux par des adultes qui n'éprouvent pas de difficultés comme par des élèves qui en rencontrent, elle ne propose pas directement de situation nouvelle pour enseigner les nombres décimaux et leur comparaison. Le scénario d'aide a en effet été élaboré principalement en articulant les résultats de recherches précédentes menées depuis plus de vingt ans en didactique des mathématiques sur ce contenu particulier. Pourtant, une partie de cette recherche porte directement sur la relation enseignement-apprentissage, elle focalise sur la composante médiative des pratiques enseignantes en proposant aux enseignants des moyens supplémentaires d'aider leurs élèves. Elle propose des modalités d'interaction précisément définies afin d'intervenir auprès de ceux qui restent en difficulté après l'institutionnalisation des procédures à mettre en œuvre pour comparer des nombres décimaux, en conjuguant simultanément le développement et l'organisation de leurs connaissances sur ces nombres.

\section{Références}

Bolon, J. (1996). Comment les enseignants tirent-ils parti des recherches faites en didactique des mathématiques? Le cas de l'enseignement des décimaux à la charnière école - collège. Thèse en didactique des mathématiques, Université Paris 5.

Brousseau, G. (1980). Problèmes de l'enseignement des décimaux. Recherches en didactique des mathématiques, 1(1), 11-59.

Brousseau, G. (1981). Problèmes de didactique des décimaux. Recherches en didactique des mathématiques, 2(1), 37-127.

Brousseau, G. (1983). Les obstacles épistémologiques et les problèmes en mathématiques. Recherches en didactique des mathématiques, 4(2), 165-198.

Brousseau, G. (1998). Théorie des situations didactiques. Grenoble: La Pensée sauvage.

Chapet-Pariès, M. (2004). Comparaison de pratiques d'enseignants de mathématiques. Recherches en didactique des mathématiques, 24(2-3), 251-284

Chevallard, Y. (1999). L'analyse des pratiques enseignantes en théorie anthropologique du didactique. Recherches en didactique des mathématiques, 19(2), 221-265.

Collet, M. (2003). Le développement du système en base 10 chez des élèves de $2^{\mathrm{e}}$ et de $3^{\mathrm{e}}$ année primaire: une étude exploratoire. Éducation et francophonie, 31(2), 218-241.

Comiti, C. et Neyret, R. (1979). À propos des problèmes rencontrés lors de l'enseignement des décimaux en classe de cours moyen. Grand N, 18, 5-20.

DeBlois, L. (1996). Une analyse conceptuelle de la numération de position au primaire. Recherches en didactique des mathématiques, 16(1), 71-127.

Dehaene, S. et Cohen, L. (1995). Towards an anatomical and functional model of number processing. Mathematical Cognition, 1, 83-120.

Douady, R. (1986). Jeux de cadres et dialectique outil-objet. Recherches en didactique des mathématiques, 7(2) 5-31. 
Douady, R. et Perrin-Glorian, M.J. (1986). Liaison école-collège. Nombres décimaux. Brochure ${ }^{\circ}{ }^{62}$. Paris: IREM de Paris 7.

Duval, R. (1995). Sémiosis et pensée humaine. Berne: Peter Lang.

Fayol, M. (1990). L'enfant et le nombre. Neuchâtel: Delachaux et Niestlé.

Grisvard, C. et Léonard, F. (1981). Sur deux règles implicites utilisées dans la comparaison de nombres décimaux positifs. Bulletin vert APMEP, 327.

Grisvard, C. et Leonard, F. (1983). Résurgence de règles implicites dans la comparaison de nombres décimaux. Bulletin vert APMEP, 340.

Hinrichs, J.V., Yurko, D.S. et Hu, J.M. (1981). Two-digit number comparison: Use of place information. Journal of Experimental Psychology, Human Perception and Performance, 7(4), 890-901.

Meljac, C. (2001). Piaget, Broca, Poincaré, Mc Closkey et quelques autres. In A. Van Hout et C. Meljac (dir.), Troubles du calcul et dyscalculies chez l'enfant (p. 118-135). Paris: Masson.

Newman, R.S. et Berger, C.F. (1984). Children's numerical estimation: Flexibility in the use of counting. Journal of Educational Psychology, 76(1), 55-64.

Neyret, R. (1979). Décimaux. Grand N, 17, 5-20.

Perret, J.-F. (1985). Comprendre l'écriture des nombres. Berne: Peter Lang.

Perrin-Glorian, M.-J. (1986). Représentations des fractions et des nombres décimaux chez des élèves de CM2 et de collège. Petit $x, 10,5-29$.

Robert, A. et Rogalski, J. (2002). Le système complexe et cohérent des pratiques des enseignants de mathématiques: une double approche. Revue canadienne de l'enseignement des sciences, des mathématiques et des technologies, 2(4), 505-528.

Robert, A. et Rogalski, J. (2005). A cross-analysis of the mathematics teacher's activity. An example in a French 10th-grade class. Educational Studies in Mathematics, 59(1-3), 269-298.

Robert, A. (2005). Quelles différences y a-t-il...? Exemples d'analyses didactiques d'exercices et d'activités d'élèves. Bulletin vert APMEP, 457, 226-238.

Roditi, E. (2000). Ordre de grandeur et multiplication des décimaux. Bulletin vert APMEP, 431, 719727.

Roditi, E. (2004). Former par la résolution de problèmes professionnels. Cahier DIDIREM n 48 . Paris: IREM de Paris 7.

Roditi, E. (2005a). Les pratiques enseignantes en mathématiques - Entre contraintes et liberté pédagogique. Paris : L'Harmattan.

Roditi, E. (2005b). L'éducation face aux théories de la construction du nombre. Spirale, 36, 37-52.

Vergnaud, G. (1990). La théorie des champs conceptuels. Recherches en didactique des mathématiques, 10(2-3), 133-170.

Vinh, B. (1966). La méthode clinique et la recherche en psychologie de l'enfant. In B. Inhelder (dir.), Psychologie et épistémologie génétique (p. 67-81). Paris: Dunod.

Vygotski, L.S. (1985). Pensée et langage. Paris: Messidor. 